\title{
Raman Imaging Approach to the Study of Ferroelectric Domains and Raman Spectra of Multiferroic Boracites
}

\author{
M.N. ILIEV ${ }^{a}$, V.G. HADJIEV ${ }^{a}$, J.ÍñIGUEZ $^{b}$ AND J. PASCUAL ${ }^{c, d}$ \\ ${ }^{a}$ Texas Center for Superconductivity, University of Houston, Houston TX 77204-5002, USA \\ ${ }^{b}$ Institut de Ciencia de Materials de Barcelona (ICMAB-CSIC), 08193 Bellaterra, Spain \\ ${ }^{c}$ Institut Català de Nanotecnologia (ICN) Edifici CM7, Campus de la UAB 08193 Bellaterra (Barcelona), Spain \\ ${ }^{d}$ Departament de Física Edifici Cn, Universitat Autònoma de Barcelona 08193 Bellaterra (Barcelona), Spain \\ The boracites with general formula $\mathrm{M}_{3} \mathrm{~B}_{7} \mathrm{O}_{13} \mathrm{X}(\mathrm{M}=$ divalent metal, $\mathrm{X}=\mathrm{Cl}, \mathrm{Br}$, I), shortly denoted as \\ $\mathrm{M}-\mathrm{X}$, are among the first known multiferroic materials. They exhibit a sequence of transitions from the high \\ temperature paraelectric cubic phase to ferroelectric orthorhombic, monoclinic, trigonal phases, and finally to a \\ monoclinic phase at low temperatures, where both ferroelectric and magnetic orders coexist. The lattice dynamics \\ of boracites has been scarcely studied with the Raman spectroscopy, the main problem with non-cubic phases \\ being the coexistence of twin variants with different crystallographic and polarization orientation. In this work, \\ on the example of $\mathrm{Co}_{3} \mathrm{~B}_{7} \mathrm{O}_{13} \mathrm{Cl}(\mathrm{Co}-\mathrm{Cl}), \mathrm{Co}_{3} \mathrm{~B}_{7} \mathrm{O}_{13} \mathrm{Br}(\mathrm{Co}-\mathrm{Br})$ and $\mathrm{Ni}_{3} \mathrm{~B}_{7} \mathrm{O}_{13} \mathrm{Br}(\mathrm{Ni}-\mathrm{Br})$ we demonstrate that \\ using the Raman microscopy imaging one can visualize the twin variants, follow their transformation through the \\ crystallographic transitions, obtain Raman spectra from untwinned domains in exact scattering configurations, \\ determine the Raman mode symmetries, and assign Raman lines to definite atomic motions. The effects of \\ structural transitions and elemental substitution are discussed in close comparison with results of ab initio \\ calculations of the phonon structure of the cubic phase.
}

PACS numbers: 78.30.-j, 77.84.Bw

\section{Introduction}

The boracites are materials with general formula $\mathrm{M}_{3} \mathrm{~B}_{7} \mathrm{O}_{13} \mathrm{X}$, shortly denoted as $\mathrm{M}-\mathrm{X}$, where $\mathrm{M}$ is divalent metal and $\mathrm{X}$ is halogen element. Their high temperature phase is cubic $(F \overline{4} 3 c)$. On cooling in most cases there is a transition to a ferroelectric orthorhombic phase $\left(P c a 2_{1}\right)$, followed by transitions to ferroelectric monoclinic $(\mathrm{Pa})$, and trigonal phases $(R 3 c)$. With further lowering of temperature, in many boracites a ferromagnetic order also takes place, which classifies them as multiferroic materials $[1,2]$. Figure 1 shows the temperature ranges of these phases for the typical boracite $\mathrm{Co}_{3} \mathrm{~B}_{7} \mathrm{O}_{13} \mathrm{Cl}(\mathrm{Co}-\mathrm{Cl})$. The crystallographic structure at room temperature for several other boracites is also indicated.

The studies of lattices dynamics of boracites have been restricted so far to several reports of 1970s on the Raman spectra of cubic and orthorhombic boracites [3-8] and a single recent report on the Raman scattering of trigonal $\mathrm{Co}-\mathrm{Cl}$ at room temperature [9]. Earlier measurements of Raman spectra of the non-cubic phases in exact scattering configurations have been hampered by the heavily twinned structure [10]. Combined with the large number of the Raman allowed modes, it has been practically impossible to determine the Raman line symmetries and to make phonon mode assignment. To a great extent this obstacle has been overcome by Iliev et al. [9]

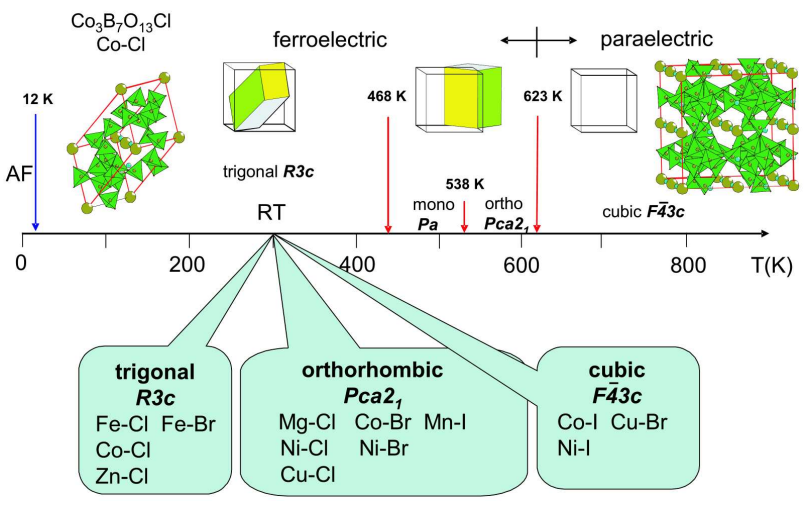

Fig. 1. Crystallographic phases of $\mathrm{Co}_{3} \mathrm{~B}_{7} \mathrm{O}_{13} \mathrm{Cl}$ $(\mathrm{Co}-\mathrm{Cl})$. The callouts show the structure of other boracites at room temperature. Boracites: $\mathrm{M}_{3} \mathrm{~B}_{7} \mathrm{O}_{13} \mathrm{X}$ or $\mathrm{M}-\mathrm{X}(\mathrm{M}=$ divalent metal, $\mathrm{X}=$ chalcogen $)$.

by Raman visualization of crystallographically distinct twin variants. This approach allows one to obtain spectra from untwinned domains in several exact scattering configurations, including those where either pure TO or pure LO modes are pronounced, and assign some of the Raman lines to definite atomic motions. In this work the Raman studies of $\mathrm{Co}-\mathrm{Cl}$ are extended to all crystal- 
lographic phases. The spectra in the orthorhombic and cubic phases are compared to those of $\mathrm{Co}-\mathrm{Br}$ and $\mathrm{Ni}-\mathrm{Br}$. The spectra in the cubic phase are analyzed in close comparison with predictions of first principles calculations of the lattice dynamics of $\mathrm{Co}-\mathrm{Cl}$.

\section{Twin variants in non-cubic boracites}

Figure 2 illustrates the different orientation of twin variants with respect to crystallographic directions of the parent cubic phase. Obviously, depending on the twin variant(s) at the laser focus spot, the scattering configuration may vary over the pseudocubic crystal surface. For this reason, the verification that the Raman spectrum is obtained from a single twin domain and determination of domains's crystallographic directions is an ultimate prerequisite for the Raman spectra analysis.

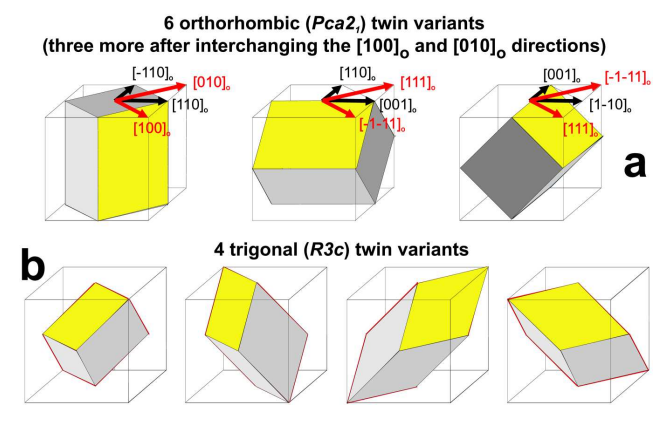

Fig. 2. Twin variants of (a) orthorhombic and (b) trigonal boracite structures. The monoclinic structure is not well established, but it is expected that the monoclinic twins are similar to the orthorhombic ones.

\subsection{Raman visualization of twin variants}

The principle of the Raman visualization of twin variants is simple, but it could be realized only after high sensitive detectors and proper software became available. It is based on the fact that the intensity of a Raman line depends on the scattering configuration, which, at fixed incident and scattered light polarizations, will be different for each of the twin variants. Provided the Raman signal is strong enough, one can collect spectra from thousands of points in the selected area, create maps of domain distribution by type and orientation, and further study separately the species of interest. An example of twin visualization on the crystal surface of trigonal $\mathrm{Co}-\mathrm{Cl}$ is shown in Fig. 3.

\subsection{Identification of crystallographic directions of trigonal twin domains (the case of $\mathrm{Co}-\mathrm{Cl}$ )}

The primitive unit cell of trigonal $\mathrm{Co}-\mathrm{Cl}$ contains two formula units ( 48 atoms) and by symmetry there are in total $23 A_{1}+47 E$ Raman modes. The atomic motions of the $A_{1}$ and $E$ modes are parallel and perpendicular to the trigonal axis, respectively. The polarization selection rules are determined by the Raman tensors
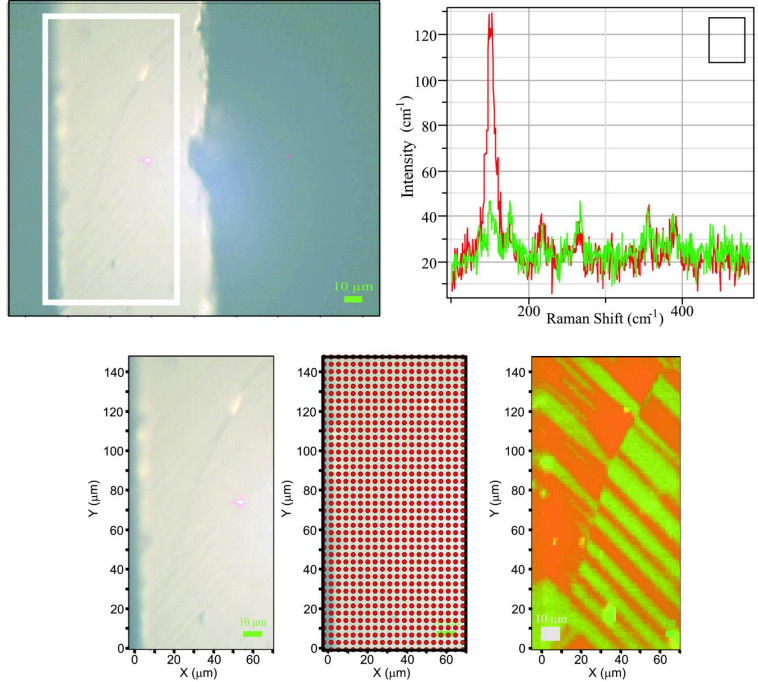

Fig. 3. Optical and Raman images of the surface of trigonal $\mathrm{Co}-\mathrm{Cl}$ single crystal at $300 \mathrm{~K}$. The Raman image has been obtained by $X Y$ scanning of the selected area by $1.5 \mu \mathrm{m}$ steps with laser spot diameter $\approx 1 \mu \mathrm{m}$. The two spectra correspond to points from green and red parts, respectively.

$$
\begin{aligned}
& A_{1}(z) \rightarrow\left[\begin{array}{lll}
a & 0 & 0 \\
0 & a & 0 \\
0 & 0 & b
\end{array}\right], \\
& E(x), E(y) \rightarrow\left[\begin{array}{ccc}
c & 0 & d \\
0 & -c & 0 \\
d & 0 & 0
\end{array}\right],\left[\begin{array}{ccc}
0 & -c & 0 \\
-c & 0 & d \\
0 & d & 0
\end{array}\right],
\end{aligned}
$$

expressed in an orthogonal coordinate system (see Fig. 4a) with $Z$-direction parallel to the trigonal axis $[001]_{\text {trig }} \|[111]_{\text {cubic }}$, and $X$ and $Y$ perpendicular to it. The facets of boracite as-grown crystals most often represent $(100)_{c},(110)_{c}$, or $(111)_{c}$ surfaces of the high temperature cubic phase (Fig. 4b, c). The projections of twin variant trigonal axes on these surfaces cross under angles of $60^{\circ}$ for $(111)_{c}$ (Fig. 4d), $90^{\circ}$ for $(100)_{c}$ (Fig. 4e), and $54.7^{\circ}$ or $70.6^{\circ}$ for $(110)_{c}$, respectively.

The determination of trigonal axis direction $Z$ is based on the reasonable assumption that some of the $A_{1}$ modes are stretching modes and their intensity in parallel scattering configuration $(H H)$ will be strongest for $H$ parallel to the projection of $Z$ and lowest for $H$ perpendicular to it. After the twin variant areas are mapped, we can stay on a single twin domain and rotate the crystal to find the direction of maximum intensity for some mode. This is illustrated in Fig. 5. The polar diagrams for the $150 \mathrm{~cm}^{-1}$ line intensity clearly show that the projections of trigonal axes of neighboring twins are mutually orthogonal and therefore the surface under investigation is $(100)_{c}$ with $[100]_{c}$ direction at $45^{\circ}$ with respect to the edge. Once the crystallographic directions of a distinct twin are found, the identification of Raman line sym- 


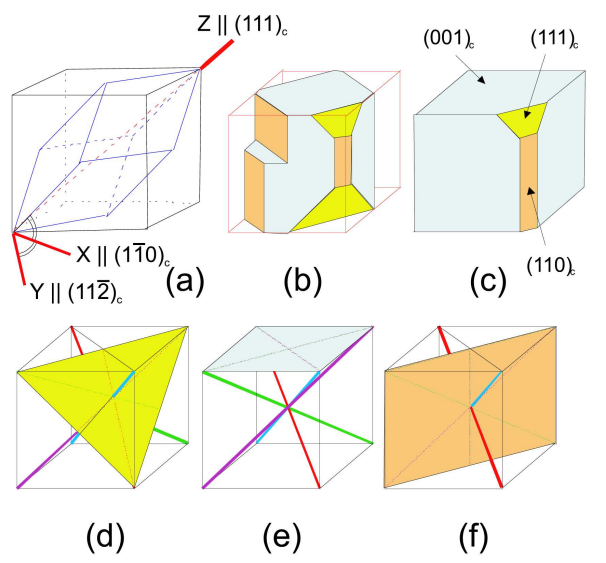

Fig. 4. (a) Coordinate system of the Raman tensors; (b), (c) most common facets of boracite as-grown crystals; (d), (e), (f) projections of the trigonal axes of twin variants on these crystal surfaces.

metries can easily be done by measuring the spectra in proper exact scattering configurations. The assignment of phonon modes to definite atomic motions, however, is not that straightforward. The problem comes from the large number of modes of given symmetry and the unavoidable uncertainty of phonon frequencies predicted by calculations of lattice dynamics. As discussed in more detail in Ref. [9], however, the stretching $A_{1}$ modes can be identified with great certainty.

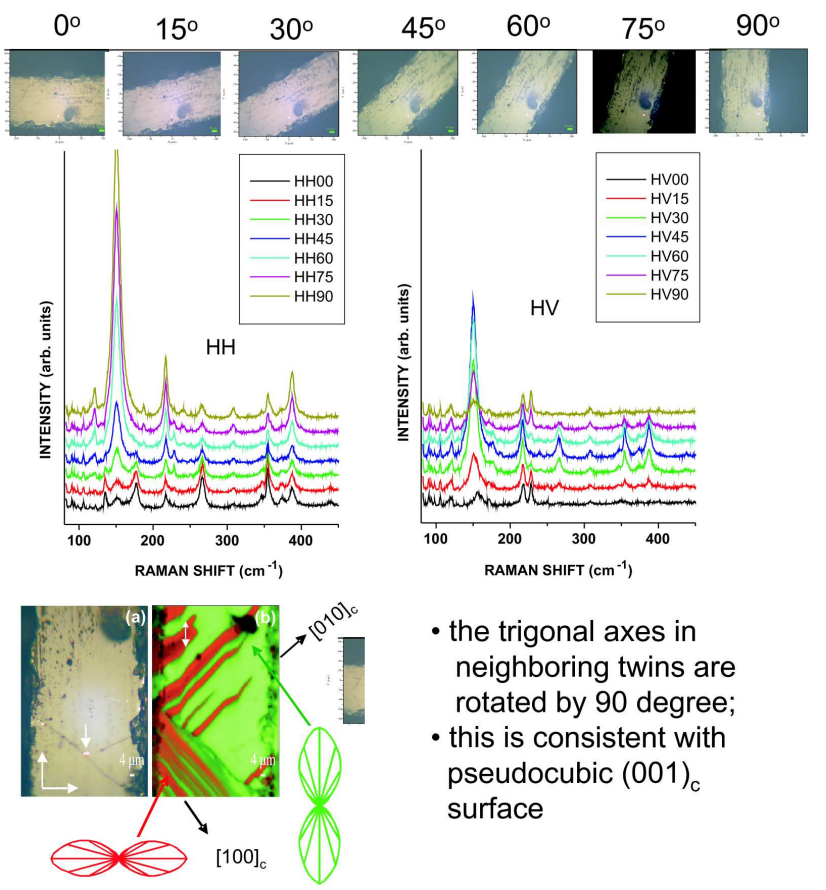

Fig. 5. Angular dependence of the Raman spectra of $\mathrm{Co}-\mathrm{Cl}$ as obtained from single twin domain with parallel $(H H)$ and crossed $(H V)$ incident and scattered light polarizations.

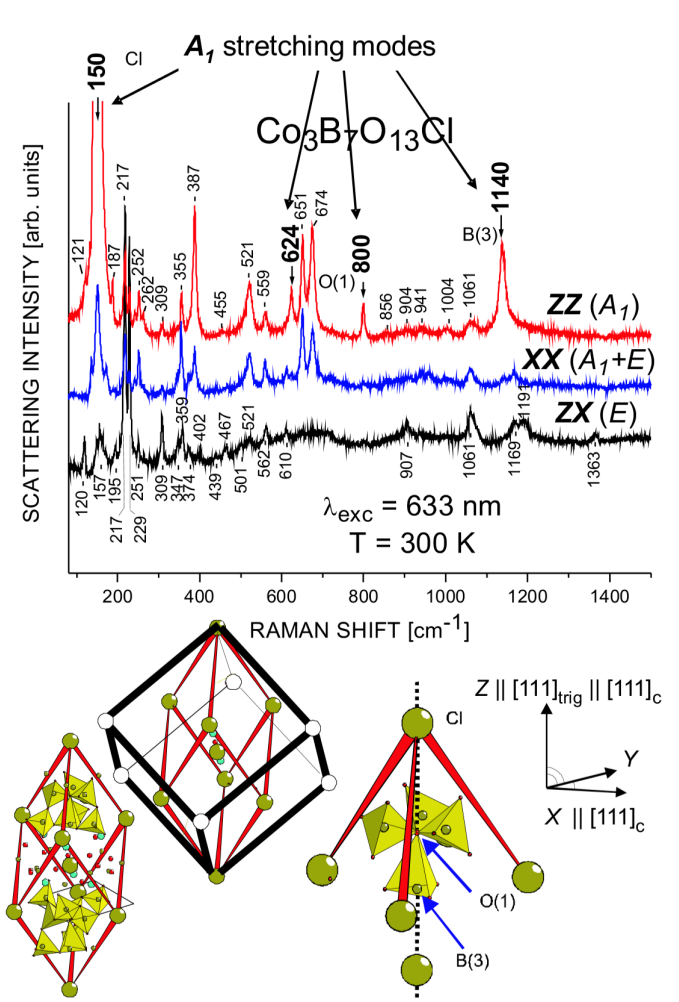

Fig. 6. $X X, Z Z$, and $X Z$ Raman spectra of trigonal $\mathrm{Co}-\mathrm{Cl}$.

Figure 6 shows the Raman spectra of trigonal $\mathrm{Co}-\mathrm{Cl}$ in pure $X X\left(A_{1}+E\right), Z Z\left(A_{1}\right)$, and $X Z(E)$ scattering configurations. Indicated are the Raman lines which most likely correspond to stretching vibrations along the trigonal axis $Z$. The atoms with nearest neighbors along $Z[\mathrm{Cl}, \mathrm{O}(1)$, and $\mathrm{B}(3)]$ are also shown. On the basis of atomic mass and bond length considerations the $150 \mathrm{~cm}^{-1}$ and $1140 \mathrm{~cm}^{-1}$ lines can be assigned, respectively, to $\mathrm{Cl}$ and $\mathrm{B}(3)$ stretchings, whereas the 624 and $800 \mathrm{~cm}^{-1}$ modes involve mainly $\mathrm{O}(1)$ vibrations.

\section{Effects of crystallographic transitions on the domain structure and Raman spectra}

Temperature dependent studies provide the opportunity to study the effect of crystallographic transitions on the domain structure and to compare the Raman spectra of different crystallographic structures. Of particular interest is the correlation between the domain structures in neighboring phases and the reversibility of domain topography upon heating or cooling through the phase transitions.

Figure 7 shows the variation with temperature of domain structure of a selected area on the quasicubic $a b$ surface of $\mathrm{Co}-\mathrm{Cl}$. This area contains a natural defect, which created domain discontinuation and verifies that the same area is mapped. As illustrated, the domain structure does not reproduce itself after the sample is heated up to its paraelectric cubic phase and cooled back to a 
ferroelectric non-cubic phase. At the orthorhombic-to-monoclinic and monoclinic-to-trigonal transitions, however, the domain structures at the both sides of transitions exhibit some similarities for reason still to be elucidated.

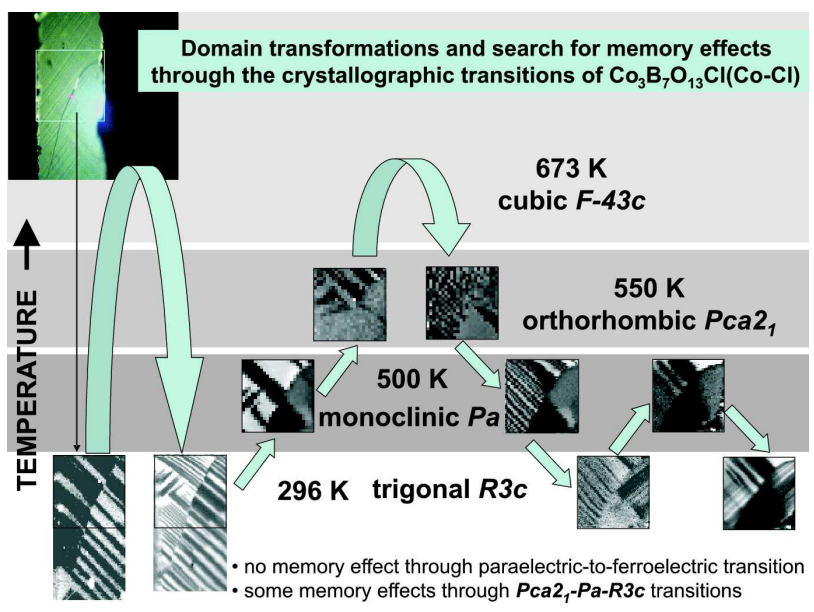

Fig. 7. Variations with temperature of the domain structure on the cubic $(100)_{c}$ surface of $\mathrm{Co}-\mathrm{Cl}$. The heating-cooling routes are indicated by arrows.

TABLE

Raman allowed phonons for the crystallographic phases of boracites.

\begin{tabular}{l|c|c}
\hline \hline \multicolumn{1}{c|}{ Structure } & $\begin{array}{c}\text { Primitive } \\
\text { cell volume }\end{array}$ & $\begin{array}{c}\text { Raman } \\
\text { modes }\end{array}$ \\
\hline cubic $F \overline{4} 3 c$ & $\mathrm{~V}$ & $4 A_{1}+10 E+20 F_{2}$ \\
orthorhombic $P c a 2_{1}$ & $2 \mathrm{~V}$ & $71 A_{1}+72 A_{2}+71 B_{1}+71 B_{2}$ \\
monoclinic $P a$ & $2 \mathrm{~V}$ & $143 A^{\prime}+142 A^{\prime \prime}$ \\
trigonal $R 3 c$ & $\mathrm{~V}$ & $23 A_{1}+47 E$
\end{tabular}

In Table there are given the number and symmetry of the Raman allowed modes for the four crystallographic phases of boracites. Although, compared to the trigonal phase, larger number of the Raman lines is expected in the temperature regions of monoclinic and orthorhombic phases, this is not confirmed by the experimental temperature-dependent spectra of $\mathrm{Co}-\mathrm{Cl}$, shown in the top part of Fig. 8. This could be explained by the relatively weak distortions differing these phases from the parent cubic one and the temperature range of phase existence. Indeed, large number of modes in the orthorhombic and monoclinic phases (160 of total 285) are due to distortion-induced splitting of $E$ and $F_{2}$ cubic modes (most of them of low or negligible intensity) additionally doubled by the twice larger primitive cell volume. If the temperature is relatively high, as is the case of ortho- and mono- $\mathrm{Co}-\mathrm{Cl}$, the thermal broadening results in overlapping of split-off components and strong decrease of maximum intensity of the resulting complex band, if still detectable. The broadening effect becomes evident if the spectra of orthorhombic phases of $\mathrm{Co}-\mathrm{Cl}$ and $\mathrm{Ni}-\mathrm{Br}$, shown in Fig. 8 are compared.

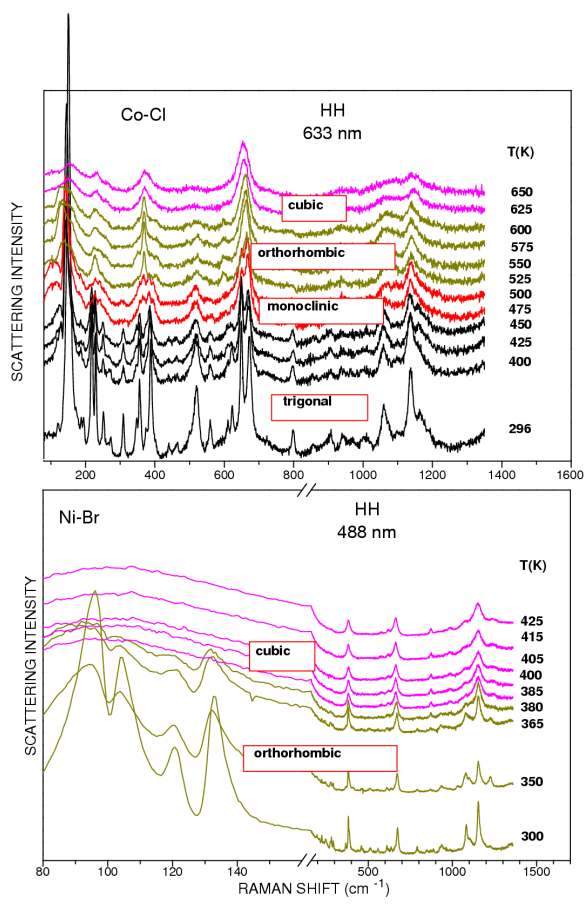

Fig. 8. Variations with temperature of the Raman spectra of $\mathrm{Co}-\mathrm{Cl}$ and $\mathrm{Ni}-\mathrm{Br}$.

As illustrated by the spectra of Fig. 8, except for some temperature shift, the center of gravity of the groups of lines, visibly related to Raman bands of the cubic phase spectra, remains practically at the same frequency through all crystallographic transitions. This is particularly true for the spectral structures above $300 \mathrm{~cm}^{-1}$ and has to be expected. Indeed, the Raman modes in the middle- and high-frequency range involve mainly vibrations of boron and oxygen in the framework of two rigid building units: symmetric $\mathrm{BO}_{4}$ and asymmetric $\mathrm{OBO}_{3}$ tetrahedra (see Fig. 9). The crystallographic transitions affect mainly the positions and bond lengths of the halogen $(\mathrm{Cl})$ and metal $(\mathrm{Co})$ atoms and to much lesser extent those of the $\mathrm{BO}_{4}$ and $\mathrm{OBO}_{3}$ tetrahedra. Therefore, most dramatic effect of phase transitions may be expected in the low frequency range, where are the modes with the dominant participation of heavier halogen and metal atoms. It is worth mentioning here that these modes are not Raman active in the cubic phase, but pronounce themselves quite strongly in the non-cubic ferroelectric phases.

\section{Phonon energies and phonon density of states calculated from first principles}

For better understanding the phonon structure of boracites, we performed first principles density functional theory (DFT) lattice dynamical calculations for the high symmetry paraelectric cubic phase of $\mathrm{Co}-\mathrm{Cl}, \mathrm{Co}-\mathrm{Br}$, and 


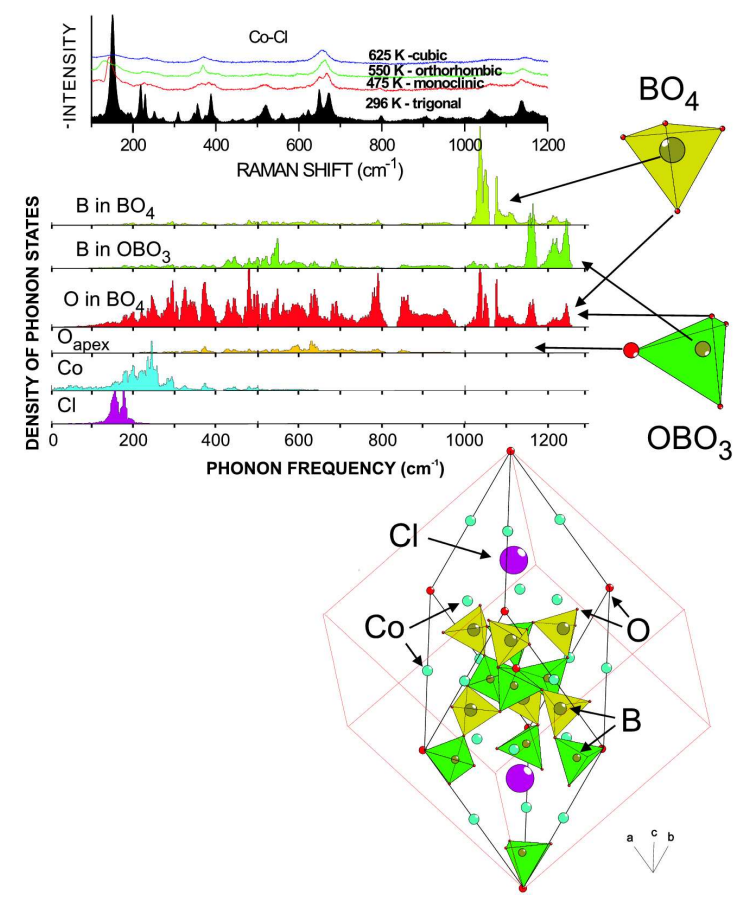

Fig. 9. Building elements of boracite structures and partial density of phonon states for nonequivalent atoms of cubic $\mathrm{Co}-\mathrm{Cl}$.

Co-I [11]. The idea was that the relatively low number of Raman phonons in the cubic phase would allow more reliable comparison to the experimental data. It was also reasonable to expect that the lattice distortions, distinguishing the different phases of a given material will only slightly affect the partial phonon density of states (PDOS), which would help in identifications of modes related to halogen and metal atoms, which are Raman forbidden in the cubic phase.

A detailed description of the DTF calculations of the lattice dynamics and their application to analysis of the Raman spectra of the cubic boracites will be given elsewhere [11]. We will only mention here that as starting parameters were the experimental cubic cell parameters and interatomic distances. After the structure has been optimized for minimum energy, the force constants have been calculated and the lattice matrix has been constructed. The PDOS has been obtained from phonon energy calculations at $10^{3} k$-points in the Brillouin zone. The so obtained partial PDOS's for all non-equivalent atoms in the cubic $\mathrm{Co}-\mathrm{Cl}$ are shown in Fig. 9.

The results of lattice dynamical calculations could be used to analyze the effect of elemental substitution. The top part of Fig. 10 shows the total (black line) and partial PDOS (colored areas) for $\mathrm{Br}$ and $\mathrm{Co}$ in $\mathrm{Co}-\mathrm{Br}$, and $\mathrm{Cl}$ and $\mathrm{Co}$ in $\mathrm{Co}-\mathrm{Cl}$. The only significant difference between the data for $\mathrm{Co}-\mathrm{Br}$ and $\mathrm{Co}-\mathrm{Cl}$ is the position of PDOS related to the halogen $(\mathrm{Br}$ or $\mathrm{Cl})$, which, as expected, scales with the corresponding atomic masses. It is also evident that except for Co, other atoms have practically no contribution in the energy range of halogen vibrations. The theoretical predictions for the cubic phase at $0 \mathrm{~K}$ can be compared to the experimental Raman spectra of the orthorhombic phases of $\mathrm{Ni}-\mathrm{Br}$ (at $296 \mathrm{~K}$ ), $\mathrm{Co}-\mathrm{Br}$ (at $296 \mathrm{~K}$ ), and $\mathrm{Co}-\mathrm{Cl}($ at $550 \mathrm{~K}$ ), shown in the bottom part of Fig. 10. It is evident from the comparison of these three spectra that the substitution of Ni for Co has only mild effect on the Raman spectra in the low frequency range, in contrast to the strong changes upon $\mathrm{Cl}$ for $\mathrm{Br}$ substitution.
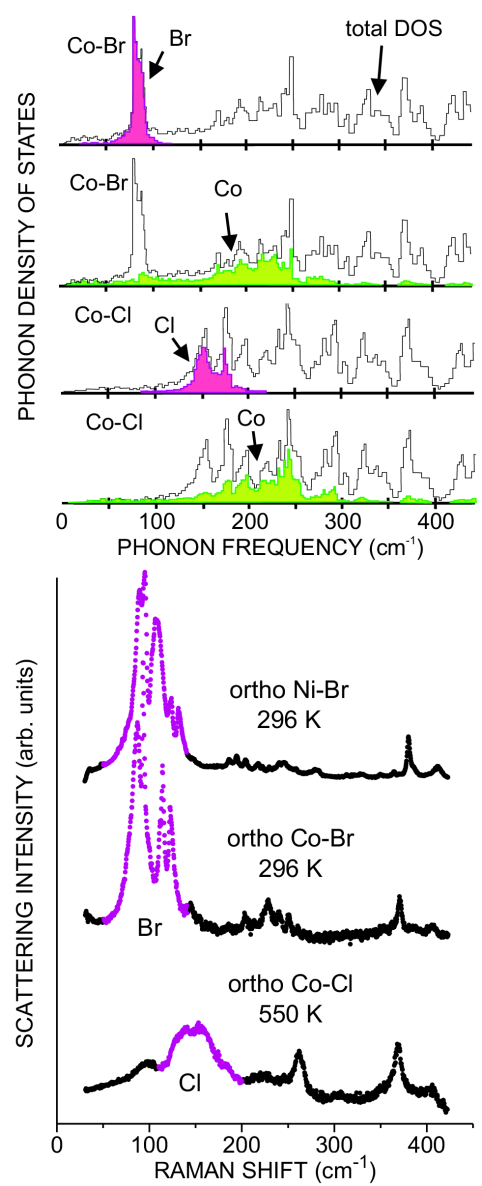

Fig. 10. (top) Total and partial density of phonon states for the cubic phase of $\mathrm{Co}-\mathrm{Br}$ and $\mathrm{Co}-\mathrm{Cl}$, as obtained by first principles calculations of the lattice dynamics [11]. (bottom) Raman spectra of orthorhombic $\mathrm{Ni}-\mathrm{Br}, \mathrm{Co}-\mathrm{Br}$, and $\mathrm{Co}-\mathrm{Cl}$.

\section{Conclusions}

It was demonstrated in this work that one of the main obstacles in the Raman studies of boracites, the uncertainty of crystallographic orientation due to coexistence of twin variants, can be overcome by their Raman visualization. This allows to obtain spectra in exact scattering configuration and follow the changes of the twin variant structure and Raman spectra through all crystallographic transitions. The consistency between the exper- 
imental results and the predictions of DFT calculations for the cubic phase is a promising first step towards better understanding of lattice dynamics and ferroelectric properties of these model materials.

\section{Acknowledgments}

We thank M.E. Mendoza for supplying us with $\mathrm{Co}-\mathrm{Cl}$, $\mathrm{Co}-\mathrm{Br}$, and $\mathrm{Ni}-\mathrm{Br}$ samples. This work was supported in part by the State of Texas through the Texas Center for Superconductivity at the University of Houston and by grant No. TK-X-1712/2007 of the Bulgarian Science Fund.

\section{References}

[1] R.J. Nelmes, J. Phys. C, Solid State Phys. 7, 3840 (1974).
[2] P. Toledano, H. Schmid, M. Clin, J.P. Rivera, Phys. Rev. B 32, 6006 (1985).

[3] D.J. Lockwood, J. Raman Spectrosc. 2, 555 (1974).

[4] D.J. Lockwood, Solid State Commun. 18, 115 (1976).

[5] A.F. Murray, D.J. Lockwood, J. Phys. C, Solid State Phys. 11, 2349 (1978).

[6] A.F. Murray, D.J. Lockwood, J. Phys. C, Solid State Phys. 11, 4651 (1978).

[7] D.J. Lockwood, Indian J. Pure Appl. Phys. 16, 268 (1978).

[8] D.J. Lockwood, Ferroelectrics 29, 19 (1980).

[9] M.N. Iliev, V.G. Hadjiev, M.E. Mendoza, J. Pascual, Phys. Rev. B 76, 214112 (2007).

[10] H. Schmid, Phys. Status Solidi 37, 209 (1970).

[11] J. Pascual, J. Ín̆iguez, M.N. Iliev, V.G. Hadjiev,J. Meen, Phys. Rev. B 79, 104115 (2009). 